12

\title{
Метод расчета нагрева замкнутых сильноточных электрических контактов импульсными токами
}

\author{
(C) М.А. Павлейно, О.М. Павлейно, М.С. Сафонов \\ Санкт-Петербургский государственный университет, физический фракультет, \\ 198504 Петергоф, Санкт-Петербург, Россия \\ e-mail: s.pavleino@yandex.ru
}

Поступило в Редакцию 25 апреля 2020 г.

В окончательной редакции 8 июня 2020 г.

Принято к публикации 26 июня 2020 г.

Предложен оригинальный метод численного расчета нагрева сильноточных электрических контактов, находящихся в замкнутом состоянии, импульсными токами, длительность которых сравнима с периодом тока промышленной частоты. Такие режимы характерны для токоведущих систем высоковольтных электрических аппаратов при коммутации нагрузок большой мощности, при возникновении аварий в линиях электропередач, при испытаниях аппаратов на стойкость к токам короткого замыкания. При построении решения считаются известными зависимости от времени протекающего тока и напряжения на контактах. Разработана итерационная процедура решения серии термоэлектрических задач, которая путем подбора на каждом временном интервале размера контактных пятен позволяет рассчитать распределение температуры в контактах и проследить за его изменением. Проведено тестирование данного метода путем сопоставления результатов расчета с экспериментальными данными, полученными для цилиндрических медных контактов при их нагреве до плавления импульсным током в широком диапазоне сил контактного нажатия. Токовые воздействия выбраны такими, что плавление происходит за максимально короткое время - в течение первой четверти периода.

Ключевые слова: электрический контакт, импульсный нагрев, численный расчет, расплывание контактного пятна.

DOI: 10.21883/JTF.2021.01.50281.148-20

\section{Введение}

Сильноточные электрические контакты входят в состав токоведущих систем различных электротехнических устройств. Наиболее распространенными из них являются высоковольтные электрические аппараты, управляющие работой электрических сетей, передающих электроэнергию от генерирующих объектов всевозможным потребителям. От надежности работы электрических аппаратов напрямую зависит качество энергоснабжения.

В процессе эксплуатации токоведущие системы электрических аппаратов подвергаются токовым воздействиям, существенно различающимся по величине, форме и длительности. Протекание токов приводит к нагреву токоведущих систем, в особенности, электрических контактов в силу того, что контакты обладают дополнительным сопротивлением по сравнению с прилегающими к ним проводниками из-за эффекта стягивания линий тока к проводящим контактным пятнам малых размеров [1]. Разрабатываемые аппараты должны успешно пройти легализационные испытания на воздействие номинальными токами и токами короткого замыкания. В процессе испытаний на стойкость контактов к току короткого замыкания контакты остаются замкнутыми в течение всего времени протекания тока, длительность которого составляет от 1 до $4 \mathrm{~s}$.
При протекании номинальных токов распределение температуры по токоведущей системе стационарно. Оно формируется за счет выделения тепла в электрических контактах и смежных проводниках и теплообмена с окружающей средой. Допустимые температуры, как правило, не превышают $100^{\circ} \mathrm{C}$, что приводит к слабой нелинейности процесса нагрева. Размеры контактных пятен практически не изменяются, зависимость от температуры электрических и тепловых контактных сопротивлений известны.

Процедуры численного расчета нагрева номинальным током в настоящее время хорошо разработаны и позволяют рассчитывать температурные поля довольно сложных устройств. Инструментом расчета в большинстве случаев является пакет численного моделирования ANSYS CFX.

Задача расчета нагрева контактов токами короткого замыкания существенно сложнее. Процесс нагрева не стационарен, температуры контактов в процессе расчета могут достигать значения температуры плавления, а элементов токоведущих систем превышать температуру размягчения. При этом в полной мере проявляются нелинейные свойства материалов: механические, электрические и тепловые.

Токи короткого замыкания имеют гармоническую и апериодическую составляющие. Величина амплитуды в первом полупериоде, называемая ударным током короткого замыкания, может существенно, почти в два раза, 
превосходить установившееся значение тока короткого замыкания. Ее значение зависит от многих факторов: фазы номинального тока, при которой происходит авария, погонных параметров линии, ее топологии, месте возникновения аварии, ее типа и параметров [2]. При наличии скачка тока в первом полупериоде особенно сильно проявляется нестационарность и нелинейность процесса нагрева. В течение короткого интервала времени, исчисляющегося единицами ms, из-за наличия контактного сопротивления происходит значительное энерговыделение в малой окрестности контактных пятен. Тепло не успевает отвестись от этой области за счет теплопроводности и практически все расходуется на нагрев пятен. В это же время удаленные части токоведущей системы изменяют свою температуру незначительно.

Процессы, протекающие в контактах в течение первого полупериода тока короткого замыкания, могут оказать существенное влияние на ход дальнейшего их нагрева и нагрева токоведущей системы в целом. В это время может произойти размягчение материала, что приводит к росту размера контактных пятен и уменьшению контактного сопротивления, что сказывается на дальнейшем нагреве. При достижении в первом полупериоде значения температуры плавления может произойти плавление замкнутых контактов, следствием чего практически всегда является их фатальная сварка, приводящая к потере работоспособности электрического аппарата [3].

Стоит отметить, что именно сложность корректного описания нагрева в первом полупериоде является принципиальным затруднением построения эффективных методов численного анализа прохождения токов короткого замыкания через замкнутые сильноточные электрические контакты [4].

В настоящей работе мы предложим способ расчета нагрева контактов импульсными токами до плавления, который позволит в значительной степени преодолеть отмеченные трудности. Но сначала проанализируем основные методы расчета импульсного нагрева контактов, существующие на сегодняшний день, отметим их основные особенности и возможные области применения.

\section{1. Метод прямого численного расчета импульсного нагрева контактов}

По своей сути задача импульсного нагрева замкнутых контактов является задачей о протекании заданного тока $I(t)$ из одного проводника в другой через одно или несколько контактных пятен, расположенных на поверхностях их соприкосновения. Форма и размер контактных пятен определяется формой контактирущих поверхностей, свойствами материала, величиной силы контактного нажатия и температурой. В более общей постановке могут рассматриваться не только сами контакты, но и представляющие интерес фрагменты токоведущих систем электрических аппаратов, включающие контакты.
С точки зрения численной реализации подобные задачи являются термоэлектромеханическими контактными задачами [5], возможность решения которых предоставляют многие пакеты численного моделирования, такие как ANSYS, COMSOL и др. С их помощью можно решать задачи нагрева токами короткого замыкания как самих контактов, так и токоведущих систем в целом. Однако при решении практических задач возникает ряд существенных затруднений, связанных со сложностью определения размеров контактных пятен и их изменения в процессе нагрева в широком диапазоне температур с необходимой точностью. Покажем на простом примере к каким ошибкам в определении температуры прямым методом расчета могут привести незначительные на первый взгляд неточности определения радиусов контактных пятен.

Рассмотрим нагрев двух медных соосно расположенных цилиндрических контактов, соединенных одним контактным пятном круглой формы, расположенном на их оси. Предположим, что радиус пятна изменяется во времени так, как показано на рис. 1, $a$ сплошной линией: сначала он постоянен и равен $a_{0}$, затем с некоторого момента времени $t_{1}$ начинается его линейный рост и выход на новое постоянное значение $1.17 a_{0}$ к моменту времени $t_{2}$. Подобное поведение радиуса, как будет показано ниже в разд. 3, осуществляется при пропускании тока с максимальным значением $29.9 \mathrm{kA}$ в первом полупериоде через цилиндрические контакты с типом контактной поверхности „сфера-плоскость“ при силе контактного нажатия 1000 N. Назовем такую реализацию изменения радиуса во времени „основной“: $a^{\text {bas }}(t)$.

При решении задачи методом прямого расчета мы получим, в силу разных причин, другую функцию, описывающую изменение радиуса. Рассмотрим несколько таких функций, расположенных близко к $a^{\text {bas }}(t)$. Пусть они немного отличаются только значением конечного радиуса пятна, а параметры $a_{0}, t_{1}$ и $t_{2}$ оставим прежними. Возьмем значения конечного радиуса равными $a_{1}=1.07 a_{0}, a_{2}=1.27 a_{0}$ (рис. $1, a$ ). Отличие конечных радиусов от $a_{\kappa}^{\text {bas }}=1.17 a_{0}$ составляет всего $\pm 10 \%$.

При известной зависимости радиуса контактного пятна от времени решение задачи нагрева контактов становится тривиальной процедурой. Это стандартная термоэлектрическая задача нагрева проводника заданной конфигурации известным током. На рис. $1, b$ приведены рассчитанные кривые нагрева контактов, т.е. зависимости от времени их максимальной температуры.

При изменении радиуса контактного пятна на 17\% из-за размягчения материала („основная“ кривая нагрева) контакты нагреваются до температуры, близкой к $1200 \mathrm{~K}$, что ниже температуры плавления.

Если при вычислениях методом прямого численного расчета допущена неточность в вычислении конечного радиуса всего на $\pm 10 \%$, то в результате получаются две принципиально отличающиеся друг от друга кривые нагрева - верхняя и нижняя кривые на рис. $1, b$. В одном случае будет сделан неправильный вывод о 

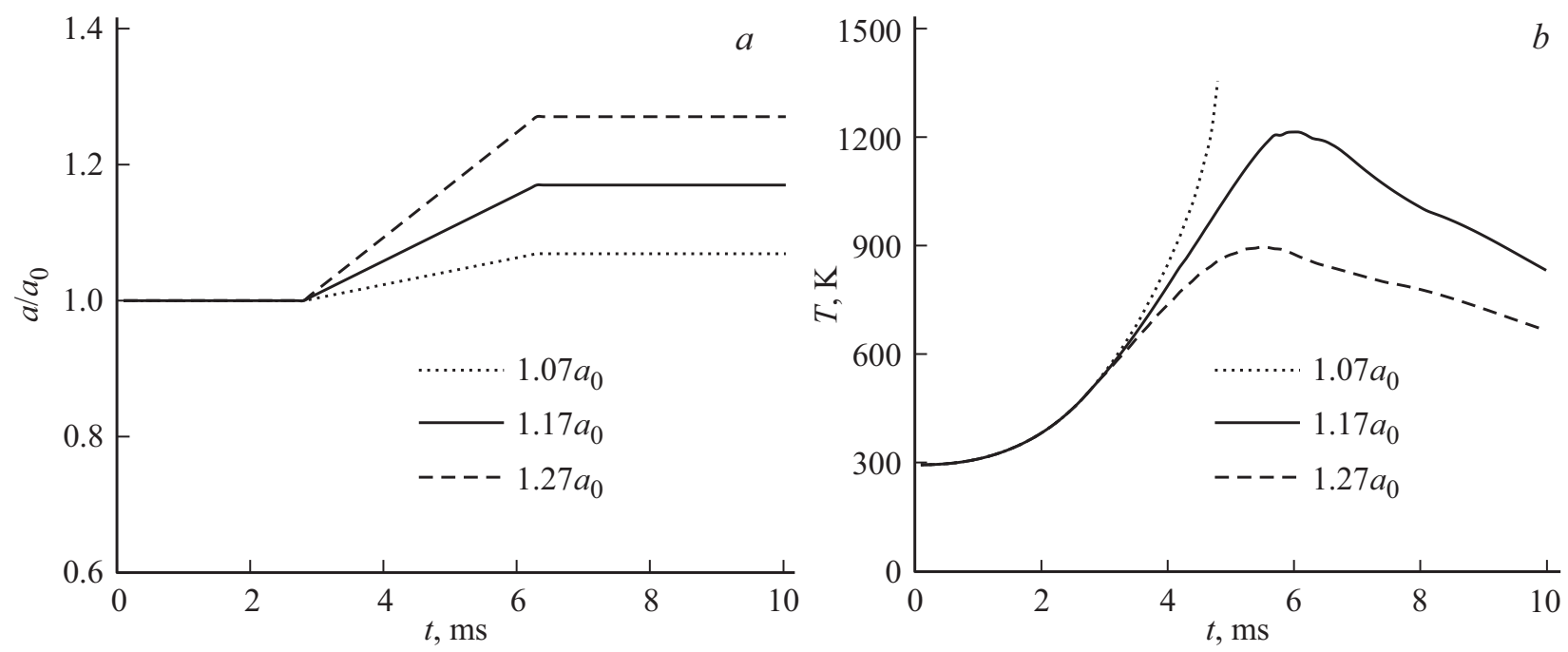

Рис. 1. Зависимость от времени радиуса контактного пятна $(a)$, зависимость от времени максимальной температуры контактов $(b)$.

плавлении контактов и, как следствие, их фатальной сварке. В другом случае будет получен результат, говорящий о большом запасе по температуре (более $350 \mathrm{~K}$ ) по сравнению с температурой плавления, что также далеко от реальной картины.

Таким образом, для решения практических задач нагрева контактов ошибка в определении радиуса контактного пятна в процессе его размягчения даже в $10 \%$ может оказаться недопустимо большой ввиду сильной зависимости динамики нагрева от этого параметра. Гарантировать же столь высокую точность решения динамической механической контактной задачи при нагреве в широком диапазоне температур, вплоть до плавления, практически невозможно. На это есть несколько причин, две из которых выделим особо.

Во-первых, следует сказать, что как в открытых источниках информации, так и в коммерческих базах данных достаточно трудно найти исчерпывающие данные о механических свойствах материала в широком диапазоне температур до плавления даже для технически чистых металлов, не говоря уже о сплавах и композитных материалах. Это связано в том числе и с тем, что указанные свойства в сильной степени зависят не только от химического состава материала, но и от технологии его производства.

Это существенно затрудняет применение методов прямого численного расчета импульсного нагрева электрических контактов для решения прикладных задач, не позволяя гарантировать требуемый уровень соответствия результатов механических расчетов реальной ситуации, что, в свою очередь, может повлечь за собой недопустимо низкий уровень точности расчета тепловых полей.

Во-вторых, принципиальным источником ошибки расчета поведения контактных пятен является изменение силы контактного нажатия при протекании токов короткого замыкания, которое точно определить и учесть при расчетах крайне сложно. Причин изменения силы нажатия несколько, и они во-многом зависят от конструкции контактов и могут проявляться одновременно. Они могут быть вызваны размягчением материала самих контактов при нагреве, что приводит к взаимному „проседанию“ контактов и снижению воздействия внешних пружин поджатия, могут нагреваться и пластически деформироваться ламели цанговых соединений и т.д.

Но главной причиной изменения силы при протекании токов короткого замыкания является воздействие ударного тока, величина которого для электрических аппаратов среднего класса напряжения может исчисляться десятками kA. При таких уровнях тока, протекающего через все элементы токоведущих систем, включая контакты, создается импульсное магнитное поле большой амплитуды, которое вызывает ударное силовое воздействие на электрические контакты и смежные с ними элементы токоведущих систем, которые механически с ними связаны, некоторые из них - жестко.

Таким образом, причинами возникновения дополнительной ударной силы, действующей на контакты, являются не только появляющееся в результате короткого замыкания магнитное поле в месте их расположения (а оно создается не только токами, протекающими через сами контакты, но и токами, текущими по весьма удаленным, но протяженным проводникам, например, внешним шинам), но и механическое воздействие на контакты соседних элементов токоведущих систем, находящихся под ударным воздействием того же поля.

Корректно рассчитать и учесть воздействие перечисленных факторов на изменение силы контактного нажатия - трудоемкая, комплексная расчетная задача. Она практически никогда не решается в полном объеме при расчете импульсного нагрева контактов с использованием прямого метода анализа. Это, наряду с отсутствием исчерпывающих данных о механических свойствах материала контактов, не позволяет гарантиро- 
вать требуемую точность решения механической части задачи для получения достоверных данных по нагреву.

В заключение по методу прямого численного расчета нагрева контактов токами короткого замыкания можно сказать следующее. Этот метод является самодостаточным (не требует наличия каких-либо результатов предварительных измерений). Он является хорошим способом для решения модельных задач, когда свойства материала и изменение силы контактного нажатия во времени считаются известными. Метод может оказаться весьма эффективным, если рассматриваются такие режимы, при которых температура размягчения в окрестности контактных пятен не достигается.

Однако при решении прикладных задач данным методом в более широком диапазоне температур могут возникать недопустимо большие ошибки по определению нагрева контактов и элементов токоведущих систем. Причем оценить величину возможных ошибок заранее (на стадии проектирования изделий без возможности экспериментальной верификации) чрезвычайно сложно.

\section{2. Метод, основанный на использовании $V-T$-соотношения}

Особенностью расчетной модели в данном случае является то, что наличие контактов учитывается их интегральными характеристиками - электрическим $R_{c}$ и тепловым $R_{T}$ контактными сопротивлениями. В модели это отражается построением в местах контактов сетки из плоских термоэлектрических контактных элементов, в свойствах которых закладываются значения величин $R_{c}$ и $R_{T}$ как функции температуры. Связь между электрическим и тепловым контактными сопротивлениями устанавливается с учетом закона Видемана-ФранцаЛоренца и имеет вид

$$
R_{T}(T)=\frac{R_{c}(T)}{L T}
$$

где $L-$ постоянная Лоренца.

Изменение контактного сопротивления $R_{c}$ в процессе нагрева происходит по двум причинам - из-за увеличения удельного сопротивления материала с ростом температуры и из-за увеличения размеров контактного пятна при нагреве выше температуры размягчения. Оба эти фактора приближенно учитываются в расчетах.

Зависимость величины $R_{c}$ от температуры контактного пятна при неизменном его размере имеет вид [1]:

$$
R_{c}\left(T_{c}\right)=R_{c 0}\left(1+\frac{2}{3} \alpha\left(T_{c}-T_{0}\right)\right),
$$

где $R_{c 0}$ - значение сопротивления при начальной температуре $T_{0}$. Коэффициент $2 / 3$, введенный Хольмом, отражает факт неравномерности нагрева контактной окрестности. Следует отметить, что выражение (2) дает хорошую оценку зависимости контактного сопротивления от температуры при нагреве контактного пятне не более, чем на $300^{\circ} \mathrm{C}[6]$.



Рис. 2. Электрические контакты, соединенные одним контактным пятном.

Величина контактного сопротивления $R_{c 0}$ измеряется до пропускания тока, а также после токового воздействия и остывания контактов $R_{c 1}$. Если в процессе протекания тока была превышена температура размягчения материала, то размер пятна увеличивается, а контактное сопротивление становится меньше: $R_{c 1}<R_{c 0}$.

В рассматриваемом методе расчета делается предположение, что размер контактного пятна и соответственно значение контактного сопротивления изменяются мгновенно при достижении контактным пятном температуры размягчения материала. Таким образом, используется следующее выражение, связывающее значение контактного сопротивления с температурой контактного пятна:

$$
R_{c}\left(T_{c}\right)=\left\{\begin{array}{ll}
R_{c 0}\left(1+\frac{2}{3} \alpha\left(T_{c}-T_{0}\right)\right), & T_{c}<T_{p} \\
R_{c 1}\left(1+\frac{2}{3} \alpha\left(T_{c}-T_{0}\right)\right), & T_{c} \geq T_{p}
\end{array} .\right.
$$

Так как сами контактные пятна и их окрестности фактически исключены из расчетной модели, информацию об изменении во времени $T_{c}$ в данной методике получают косвенным способом, используя так называемое $V-T$-соотношение. В работах $[7,8]$ была установлена связь между параметрами электрического и теплового полей контактов, которая позволяет определить температуру контактных пятен. Был исследован случай протекания постоянного тока через электрические контакты, соединенные одним контактным пятном, боковая поверхность которых теплоизолирована от окружающей среды (рис. 2). В этом случае эквипотенциальные поверхности являются также изотермическими. 

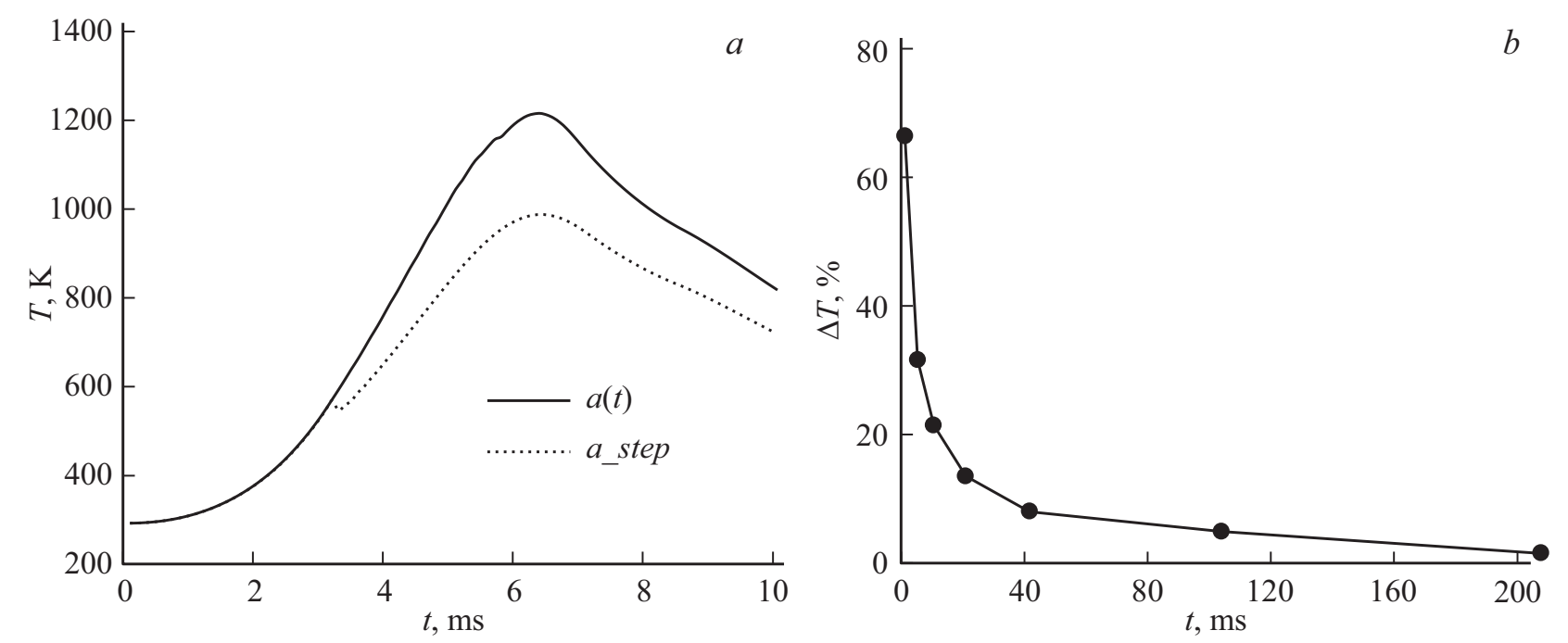

Рис. 3. Зависимость от времени максимальной температуры контактов при плавном и скачкообразном изменении радиуса контактного пятна $(a)$, погрешность определения температуры контактного пятна с помощью $V-T$-соотношения при разной длительности нагрева $(b)$.

Была установлена связь температуры контактного пятна с температурами $T_{1}$ и $T_{2}$ изотермических поверхностей $S_{1}$ и $S_{2}$, произвольно расположенных вблизи контактного пятна по разные стороны относительно его поверхности, и разностью потенциалов между ними $U_{12}$ :

$$
T_{c}=\sqrt{\frac{U_{12}^{2}}{4 L}+\frac{1}{2}\left(T_{1}^{2}+T_{2}^{2}\right)+\frac{L}{4 U_{12}^{2}}\left(T_{1}^{2}-T_{2}^{2}\right)} .
$$

Это соотношение используется для оценки текущего значения температуры контактного пятна и соответственно значения контактных сопротивлений.

Процедура построения численного решения нагрева контактов и элементов токоведущей системы следующая $[4,9,10]$. Решается переходная задача нагрева модели заданным током. На каждом временном шаге решения с помощью $V-T$-соотношения (4) оценивается температура контактного пятна. Эта величина используется для уточнения текущего значения величины электрического контактного сопротивления, согласно (3), и теплового контактного сопротивления согласно (1). Соответствующие изменения вносятся в параметры поверхностных контактных элементов, и задача решается на следующем временном интервале.

Проанализируем основные источники погрешности при использовании данного метода и оценим возможность его применения для решения задач нагрева токоведущих систем токами короткого замыкания. Начнем с предположения о скачкообразном характере изменения контактного сопротивления при достижении температуры размягчения. Авторы [9] подчеркивают, что такая модель учета размягчения контактного пятна является упрощенной и применяется по причине отсутствия сведений о динамике процесса размягчения при импульсном нагреве.
Применение такого упрощенного подхода к описанию изменения контактного сопротивления, вызванного размягчением, может привести к существенно заниженным оценкам температуры контактного пятна, что, в свою очередь, может дать ложные сведения об отсутствии плавления контактной области и фатальной сварки контактов при анализе результатов численных расчетов.

В рассмотренном выше примере нагрева контактов (рис. 1) время, в течение которого происходит размягчение контактной области и увеличение радиуса контактного пятна, составляет около $3 \mathrm{~ms}$, что сравнимо с длительностью полупериода протекающего тока. На рис. 3, $a$ приведены кривые нагрева контактного пятна, вычисленные предлагаемым нами методом итерационного подбора площади контактного пятна (см. разд. 3) с учетом реально протекающего изменения размера пятна (верхняя кривая), и в предположении мгновенно изменяющегося размера пятна (нижняя кривая). Из их сравнения следует, что приближение мгновенного изменения радиуса контактного пятна приводит к двум ошибкам. Во-первых, к заниженной оценке максимальной температуры, тогда как для практических задач лучше иметь оценку с другой стороны - гарантировать, что температура не будет больше рассчитанной. Во-вторых, к немонотонному росту температуры при нагреве.

Еще одним источником ошибки расчетов данным методом является применение (4) для определения температуры контактного пятна. Оно справедливо для стационарного нагрева, когда структуры теплового и электрического полей совпадают. Чем меньше длительность нагрева, тем больше неточность определения температуры пятна будет давать (4), так как при этом возрастает расхождение конфигурации электрического 
и теплового полей по сравнению со стационарным случаем.

Основываясь на [11], приведем зависимость погрешности определения $T_{c}$ с помощью (4), располагая точки $A_{1}$ и $A_{2}$, между которыми измеряется разность потенциалов $U_{12}$ и температуры $T_{1}$ и $T_{2}$, на расстоянии 10 радиусов контактного пятна от его поверхности для временного интервала от 1 до $200 \mathrm{~ms}$ (рис. 3, $b$ ).

Начиная с третьего периода тока короткого замыкания величина ошибки приемлемая - менее 10\%. Однако в течение первой четверти периода, когда происходит наиболее быстрый рост температуры и, собственно, размягчение контактного пятна, ошибка большая и лежит в пределах 30-70\%.

Таким образом, использование $V-T$-соотношения для оценки времени начала расплывания контактного пятна может дать недостаточно достоверные результаты.

Проведенное сравнение результатов расчета рассматриваемым методом нагрева реальных конструкций сильноточных контактов токами короткого замыкания в том числе в присутствии апериодической составляющей с результатами измерения температуры, проводимых с помощью малоинерционных термодатчиков и тепловизоров $[4,9]$, показал довольно хорошее совпадение. Однако сопоставление температур проводились на значительном удалении от мест непосредственного контакта и при перегревах, незначительно превышающих температуру размягчения материала контактов и элементов токоведущих систем.

К преимуществам метода следует отнести отсутствие необходимости решения механической контактной задачи для определения размера и формы контактных пятен. Информация о величинах контактных сопротивлений $R_{c 1}$ и $R_{c 2}$ берется из результатов измерений, что приближает расчетную модель к реальной ситуации. Однако сам процесс изменения размера контактных пятен и соответственно изменения контактного сопротивления учитывается весьма приближенно.

Метод может быть использован для расчета нагрева токоведущих систем, исключая нагрев самих контактных пятен. При этом достигается приемлемая точность расчета нагрева вне областей контактов.

\section{3. Метод расчета нагрева контактов, основанный на итерационном подборе размера контактного пятна}

Как уже было сказано, наибольшие затруднения при исследовании процесса нагрева электрических контактов токами короткого замыкания возникают при расчете температуры контактных пятен в течение первого полупериода тока, когда за единицы ms контактная область может быть нагрета до температуры плавления. Корректно описать этот процесс, используя описанные выше методы, затруднительно.
Рассмотрим возможности предлагаемого нами альтернативного способа проведения расчетов, который позволяет детально описать динамику нагрева окрестности контактных пятен и оценим степень достоверности получаемых результатов путем сравнения результатов расчета с экспериментальными данными. Опишем и протестируем данный способ расчета на примере цилиндрических медных контактов, соединенных одним контактным пятном круглой формы радиуса $a_{0}$.

Предположим, что при нагреве контактов форма пятна остается той же, а изменяется лишь его радиус $a(t)$. Экспериментально сняты осциллограммы протекающего тока $I(t)$, контактного напряжения $U_{c}(t)$, измерена величина контактного сопротивления перед прохождением импульса тока. Известны зависимости от температуры удельного сопротивления, теплопроводности, теплоемкости, плотности материала контактов.

Решение задачи импульсного нагрева осуществляется следующим образом. Исходя из известного значения контактного сопротивления, определяется начальный радиус контактного пятна $a_{0}$. Это можно сделать, исходя из хольмовского приближения, если границы контактов достаточно удалены от контактного пятна, либо численно [12]. В последнем случае путем решения серии задач определяется такое значение радиуса, которое обеспечивает значение контактного сопротивления, равное измеренному.

Далее решается нестационарная нелинейная термоэлектрическая задача о нагреве контактов током известной величины $I(t)$. Система уравнений, описывающая данный процесс, имеет вид [3]:

$$
\begin{gathered}
\rho C \frac{\partial T}{\partial t}-\nabla(\kappa \nabla T)=\frac{1}{\sigma} j^{2}, \\
\nabla^{2} \varphi=0, \\
\mathbf{E}=-\nabla \varphi, \\
\mathbf{j}=\sigma \mathbf{E},
\end{gathered}
$$

где $T$ - температура, $j$ - плотность электрического тока, $\varphi$ - электрический потенциал, $E-$ напряженность электрического поля. При решении задачи учитывается зависимость теплопроводности $k$, теплоемкости $C$, плотности $\rho$ и электропроводности $\sigma$ материала от температуры. На одной торцевой поверхности контактной системы задается нулевой потенциал, на другой величина протекающего тока. На внешней поверхности контактов задается условие адиабаты, что допустимо при длительности нагрева менее $1 \mathrm{~s}$ [13].

Решение проводится итерационно. Последовательно на каждом шаге по времени $t_{k}$ задается ток $I\left(t_{k}\right)$ и подбирается такое значение радиуса контактного пятна, которое обеспечивает заданную близость расчетного значения напряжения на контактах $U_{c}^{\text {calc }}\left(t_{k}\right)$ к экспериментально измеренному $U_{c}\left(t_{k}\right)$. Исключение в указанной процедуре составляет первый шаг, для которого радиус контактного пятна определен: $a\left(t_{1}\right)=a_{0}$, т. е. подбором 



Рис. 4. Осциллограмма тока $(a)$, первая полуволна напряжения (сплошная кривая) и расчетная зависимость напряжения от времени $(b)$, зависимость радиуса контактного пятна от времени $(c)$ и зависимость максимальной температуры контактного пятна от времени $(d)$ для реализации с амплитудой тока в первой полуволне $17.4 \mathrm{kA}$.

функции $a(t)$ мы приближаем расчетную кривую $U_{c}^{\text {calc }}(t)$ к экспериментальной $U_{c}(t)$.

В результате вычислений в любой момент времени будет определено распределение в контактной области потенциала электрического поля $\varphi(x, y, z, t)$, температуры $T(x, y, z, t)$ и производных величин - напряженности электрического поля, плотности тока, распределение тепловых потоков. Также становится известным изменение радиуса контактного пятна при нагреве $a(t)$.

Проиллюстрируем применение данного метода расчета на трех характерных примерах: нагрев контактов до температуры выше температуры размягчения, но значительно ниже температуры плавления, нагрев до температуры, близкой к плавлению, и нагрев, при котором наблюдается интенсивное плавление контактной области.

Первому случаю соответствует протекание тока через цилиндрические контакты с типом контактных поверх- ностей „сфера-плоскость“ при величине силы нажатия $1000 \mathrm{~N}$ и амплитуде первой полуволны $17.4 \mathrm{kA}$. Осциллограмма тока приведена на рис. $4, a$. На рис. $4, b$ представлена осциллограмма первой полуволны напряжения (сплошная кривая) и расчетная зависимость напряжения от времени, полученная подбором зависимости радиуса контактного пятна от времени (рис. 4,c) по описанному выше алгоритму. Расчетные точки приведены с прореживанием, реальный шаг по времени составлял $0.1 \mathrm{~ms}$. Сопоставляя полученную зависимость $a(t)$ с кривой нагрева контактного пятна (рис. 4, $d$ ), можно сказать, что расплывание пятна началось при температуре около $500 \mathrm{~K}$, радиус в процессе нагрева увеличился на $14 \%$ за $4 \mathrm{~ms}$. Максимальная температура контактного пятна была достигнута в момент времени $6 \mathrm{~ms}$, она составила $855 \mathrm{~K}$.

Во втором случае через контакты протекал ток с максимальным значением $29.9 \mathrm{kA}$ в первом полупериоде. 



Рис. 5. Осциллограмма тока $(a)$, первая полуволна напряжения (сплошная кривая) и расчетная зависимость напряжения от времени $(b)$, зависимость радиуса контактного пятна от времени $(c)$ и зависимость максимальной температуры контактного пятна от времени $(d)$ для реализации с амплитудой тока в первой полуволне $29.9 \mathrm{kA}$.

Начальная сила контактного нажатия была равна $1000 \mathrm{~N}$. На рис. 5 приведены осциллограммы тока и напряжения и результаты расчета изменения радиуса контактного пятна и изменения во времени максимальной температуры контактов.

Как и в предыдущем случае, расплывание контактного пятна началось при температуре около $500 \mathrm{~K}$, радиус увеличился на $17 \%$ за время $3.8 \mathrm{~ms}$. Максимальная температура контактного пятна в данном случае приблизилась к температуре плавления. Она превысила значение $1200 \mathrm{~K}$ в момент времени $6.2 \mathrm{~ms}$.

Рассмотрим третий пример расчета нагрева контактов (рис. 6), когда величина пропускаемого тока была достаточной для того, чтобы уже в течение первой четверти периода произошло плавление контактов. Величина тока была равна $22 \mathrm{kA}$ в первом максимуме, сила контактного нажатия равнялась $500 \mathrm{~N}$. На осциллограмме напряжения видна особенность при $t=2.15 \mathrm{~ms}$. В это время ток все еще продолжает расти, а напряжение начинает уменьшаться. В [3] показано, что такая особенность связана с началом плавления контактной области, при котором контактное сопротивление заметно уменьшается из-за роста размеров контактного пятна, вызванного плавлением. Рассчитанное значение температуры в этот момент времени отличается от температуры плавления меди менее чем на $3 \%$.

Оценим точность расчета нагрева контактов предложенным методом. Экспериментально мы можем зафиксировать время начала плавления контактного пятна, как это было сделано в последнем примере. Тем самым можно определить, в какой момент контактное пятно нагрелось до температуры плавления $T_{\text {melt }}=1356 \mathrm{~K}$ (для меди) и сравнить это значение с температурой, полученной в расчете $T_{\text {calc }}$ в тот же момент времени. По величине $\Delta=\left|T_{\text {melt }}-T_{\text {calc }}\right| / T_{\text {melt }} 100 \%$ можно судить о погрешности расчетов. На рис. 7 приведена гистограм- 

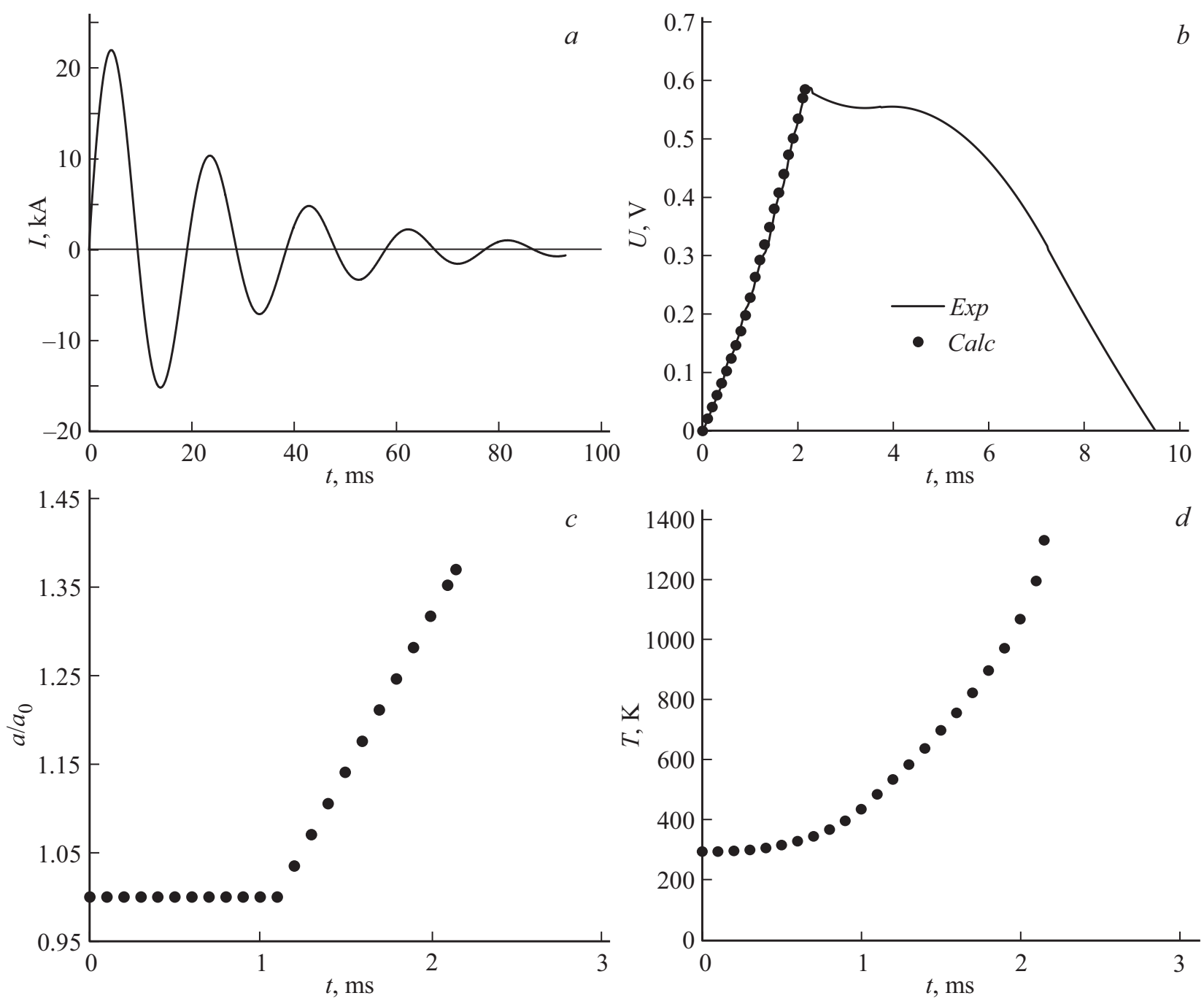

Рис. 6. Осциллограмма тока $(a)$, первая полуволна напряжения (сплошная кривая) и расчетная зависимость напряжения от времени $(b)$, зависимость радиуса контактного пятна от времени $(c)$ и зависимость максимальной температуры контактного пятна от времени $(d)$ для реализации с амплитудой тока в первой полуволне $22 \mathrm{kA}$.

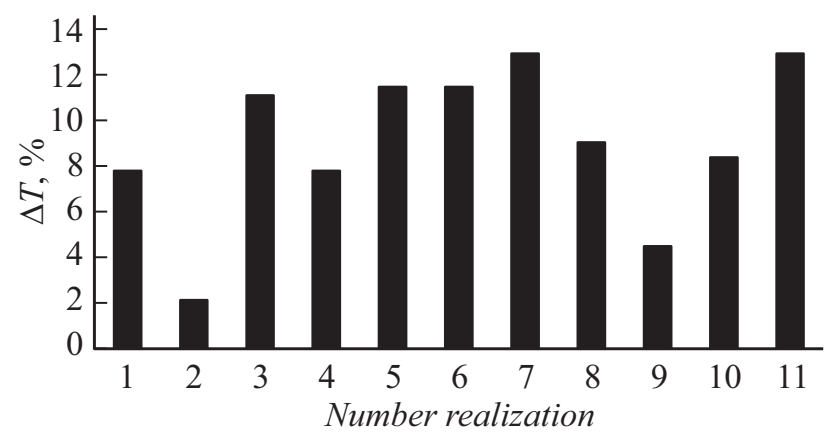

Рис. 7. Гистограмма ошибки расчета температуры в момент начала плавления.

ма ошибки определения температуры для одиннадцати реализаций такого режима в широком диапазоне сил контактного нажатия. Первые три из них соответствуют силе контактного нажатия $100 \mathrm{~N}$, три следующие - силе $500 \mathrm{~N}$, реализации № 8 и № 9 - силе $1000 \mathrm{~N}$ и две последние - сил $2500 \mathrm{~N}$.

Во всем рассмотренном диапазоне сил контактного нажатия погрешность определения температуры плавления контактов не превысила $15 \%$.

\section{Заключение}

Предложенный метод расчета импульсного нагрева контактов позволяет с достаточно высокой точностью проводить расчет нагрева электрических контактов в широком диапазоне температур, вплоть до температуры плавления, и в широком диапазоне сил контактного нажатия. Тестирование метода было проведено для наиболее трудного для расчетов режима нагрева контактов, когда температура плавления достигается уже в течение первой четверти периода импульса тока. При этом в 
окрестности контактных пятен тепловое поле характеризуется наибольшими градиентами температуры и, как следствие, реализуются наибольшие градиенты электрических, тепловых и механических свойств материала контактов.

Он может использоваться как инструмент для исследования поведения тепловых и электрических нестационарных полей при импульсном нагреве контактов. Данный метод позволяет описать процесс расплывания контактных пятен из-за размягчения материала и связанное с этим изменение величины контактного сопротивления. Полученная информация может быть использована для существенного уточнения расчетных моделей, основанных на использовании $V-T$-соотношения.

\section{Конфликт интересов}

Авторы заявляют, что у них нет конфликта интересов.

\section{Список литературы}

[1] Р. Хольм. Электрические контакты (ИИЛ, М., 1961) $464 \mathrm{c}$.

[2] Ю.А. Куликов. Переходные прочессы в электрических системах (НГТУ, Новосибирск, 2006) 284 с.

[3] О.М. Павлейно. Физические особенности нагрева сильноточных электрических контактов: Дис. канд. техн. наук. (СПб, 2015) $48 \mathrm{c}$.

[4] T. Israel, S. Schlegel, S. Grossmann, T. Kufner, G. Freudiger. IEEE Holm Conf. Electr. Contacts, Albuquerque, NM, 254 (2018). DOI: 10.1109/HOLM.2018.8611641

[5] S. Takalkar Atul, M.C. Lenin Babu. ICACCI. 2131 (2016). DOI: 10.1109/ICACCI.2016.7732367

[6] А.М. Залесский. Основы теории электрических аппаратов (Высш., шк., М., 1974) 184 с.

[7] F. Kohlrausch. Ann. Phys., 306 (1), 132 (1900).

[8] J.A. Greenwood, J.B. Williamson. P. Proc. R. Soc. Lond. Math. Phys. Eng. Sci., 246 (1244), 13 (1958).

[9] T. Israel, M. Gatzsche, S. Schlegel, S. Großmann, T. Kufner, G. Freudiger. IEEE Holm Conf. Electr. Contacts, 40 (2017). DOI: 10.1109/HOLM.2017.8088061

[10] M. Gatzsche, N. Luecke, S. Großmann, T. Kufner, G. Freudiger. IEEE Transactions on Components, Packaging and Manufacturing Technol., 7 (3), 317 (2017).

[11] Д.И. Бегаль, М.А. Павлейно, О.М. Павлейно, М.С. Сафонов, А.А. Статуя. ЖТФ, 89 (7), 1099 (2019). DOI: 10.21883/JTF.2019.07.47806.229-18

[12] О.М. Павлейно, В.А. Павлов, М.А. Павлейно. ЭОМ, 5, 56 (2010). [O.M. Pavleino, V.A. Pavlov, M.A. Pavleino. Surf. Eng. Appl. Electrochem., 64 (5), 440 (2010).]

[13] ГОСТ Р 52736-2007. Короткие замыкания в электроустановках. Методы расчета электродинамического и термического действия тока короткого замыкания (Стандартинформ, М., 2007.) 